\title{
Bilateral native lung-sparing lobar transplantation in a canine model
}

\author{
Seiichiro Sugimoto, MD, Hiroshi Date, MD, Ryujiro Sugimoto, MD, Motoi Aoe, MD, and Yoshifumi Sano, MD
}

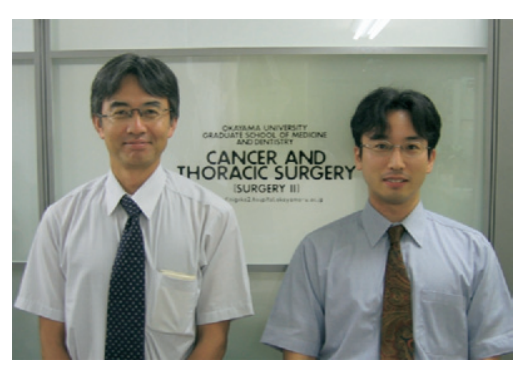

Drs Date and Sugimoto (left to right).
Objective: Bilateral living-donor lobar lung transplantation has become an accepted approach in response to the cadaveric lung donor shortage. Because only one lobe is implanted in each chest cavity, this procedure is usually confined to patients of small size. The purpose of this study was to develop a technique of bilateral native lung-sparing lobar transplantation that can be applied to large adult patients.

Methods: Bilateral native lung-sparing lobar transplantation was performed in 12 pairs of dogs. In donor animals the right middle, lower, and cardiac lobes were separated as a right graft, and the left lower lobe was separated as a left graft. In recipient animals these 2 grafts were implanted in the natural anatomic position with sparing native right upper, left upper, and middle lobes. In an acute study $(n=6)$, transplanted graft function was assessed for 3 hours after ligation of the pulmonary artery branches to the native spared lobes. In a chronic study $(n=6)$ the immunosuppressed recipients were observed for 3 weeks to assess the quality of bronchial healing and long-term pulmonary function.

Results: Morphologic adaptation of the 2 grafts was found to be excellent. All 6 animals in the acute study showed excellent pulmonary function. Five of 6 animals in the chronic study survived for 3 weeks, with excellent pulmonary function and satisfactory bronchial healing.

Conclusion: Bilateral native lung-sparing lobar transplantation was technically possible and associated with excellent pulmonary function and good bronchial healing in a canine experimental model.

$\mathrm{T}$ The persistent donor shortage in lung transplantation has resulted in increased waiting time and waiting list mortality. Bilateral living-donor lobar lung transplantation (LDLLT) was developed at the University of Southern California ${ }^{1,2}$ as a procedure for patients too ill to await cadaveric transplantation. In the existing milieu of cadaveric donor shortage in Japan, we began an LDLLT program in 1998 at our institution. ${ }^{3,4}$ This procedure can be applied only for children and small adults because a limited amount of lung tissue is implanted. In fact, we have turned down a number of patients because of unacceptable size mismatch.

To develop a new transplantation technique that can be applied to large adult patients, we recently performed canine experimental studies on unilateral double lobar lung transplantation (UDLLT) ${ }^{5,6}$ In UDLLT 2 grafts are implanted in the right hemithorax after right pneumonectomy. Although UDLLT provided excellent early pulmonary function, it was associated with a relatively high incidence of vascular and bronchial complications.

The purpose of this experimental study was to develop a technique of bilateral native lung-sparing lobar transplantation (BNSLT) that might be applied to large adult patients safely. 


\section{Abbreviations and Acronyms \\ BNSLT $=$ bilateral native lung-sparing lobar transplantation \\ LDLLT $=$ living-donor lobar lung transplantation \\ UDLLT $=$ unilateral double lobar lung transplantation}

\section{Materials and Methods}

Twelve weight-matched pairs of adult mongrel dogs $(8.5-27.0 \mathrm{~kg})$ were used for BNSLT. The dogs were randomly selected as donors or recipients. The mean weights of donor and recipient were $13.9 \mathrm{~kg}$ and $13.9 \mathrm{~kg}$, respectively.

\section{Donor Operation}

Anesthesia was induced with intramuscular ketamine hydrochloride $(10 \mathrm{mg} / \mathrm{kg})$ and atropine sulfate $(0.01 \mathrm{mg} / \mathrm{kg})$. Intravenous thiopental sodium $(2.5 \mathrm{mg} / \mathrm{kg})$ and vecuronium bromide $(0.1 \mathrm{mg} / \mathrm{kg})$ were used to facilitate endotracheal intubation. The donors were placed on a mechanical ventilator with a tidal volume of $20 \mathrm{~mL} / \mathrm{kg}$ and a positive end-expiratory pressure of $5 \mathrm{~cm} \mathrm{H}_{2} \mathrm{O}$ at a rate of 15 breaths $/ \mathrm{min}$. Anesthesia was maintained with a $0.5 \%$ inhalational halothane-oxygen mixture.

A standard median sternotomy was performed. After division of the inferior pulmonary ligaments, the pericardium was opened. The superior and inferior venae cavae were ligated and divided after systemic heparinization with sodium heparin $(200 \mathrm{U} / \mathrm{kg})$. The left atrial appendage was amputated to avoid lung congestion. The trachea was clamped, and a double-lung block was removed without flushing and cooled in cold saline solution. The right graft (right middle, lower, and cardiac lobes) and the left graft (left lower lobe) were separated from the double-lung block at the side table (Figure 1).

\section{Recipient Operation}

Anesthesia was performed as mentioned for the donor operation. Recipients were placed on a mechanical ventilator and maintained with a $0.5 \%$ inhalational halothane-oxygen mixture. A femoral artery line was inserted for measuring aortic pressure and for arterial blood gas analysis.

A right thoracotomy was performed through the fifth intercostal space, with the animals in the left lateral position. The right middle, lower, and cardiac lobes were removed, and the right upper lobe was spared. Great care was taken to avoid any air leakage from the interlobar fissure. The pulmonary artery distal to the upper lobe branches was divided between silk ligatures. Pulmonary veins, except the right upper pulmonary vein, were divided between silk ligatures placed on each venous branch at the hilum. The intermediate bronchus was divided between silk ligatures. The pericardium was opened around the pulmonary veins, and the interatrial groove was dissected to increase the length of recipient left atrium available for subsequent clamp placement.

The right graft (right middle, lower, and cardiac lobes of the donor) was implanted in the natural anatomic position (Figure 1). After placing a vascular clamp on the recipient left atrium so as not to occlude the right upper pulmonary vein, a suitable cuff

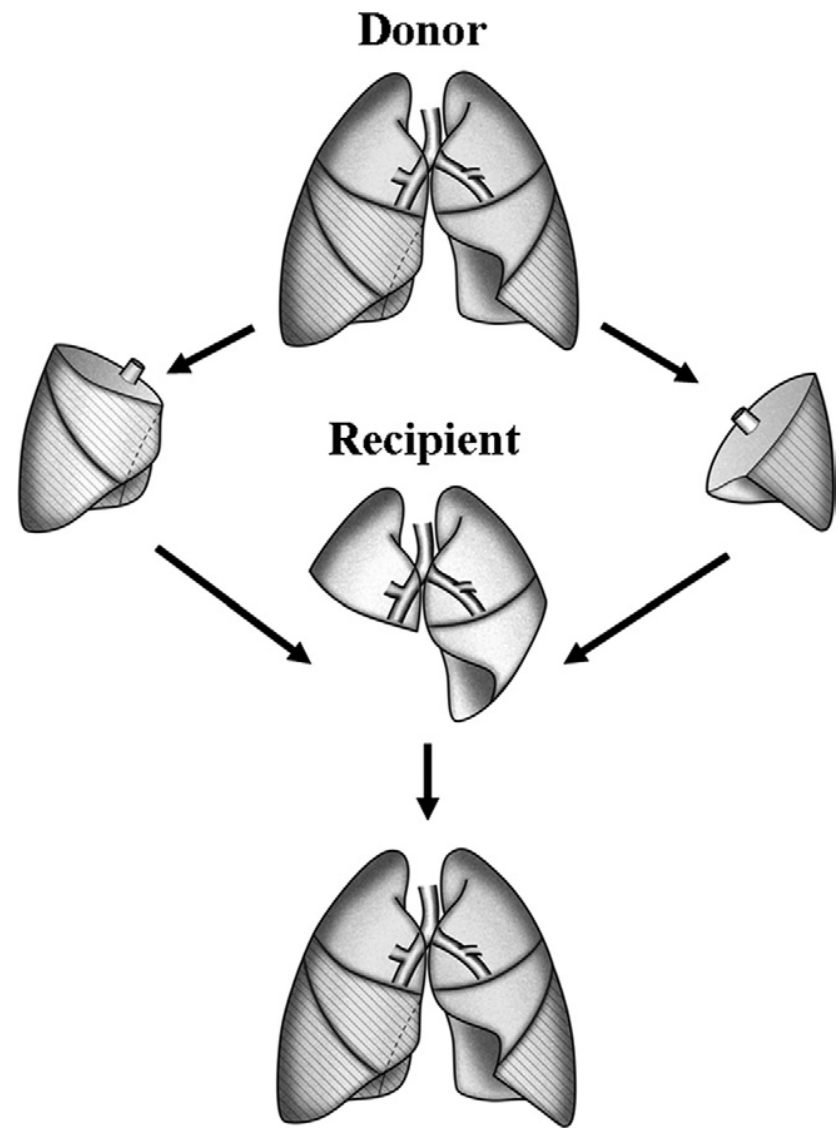

Figure 1. Bilateral native lung-sparing lobar transplantation. In the donor animal the right graft (right middle, lower, and cardiac lobes) and the left graft (left lower lobe) were separated from the double lung block. In the recipient animal both grafts were implanted in the natural anatomic position.

for the venous anastomosis was created. The donor left atrial cuff was attached to the recipient left atrial cuff by using an everting mattress suturing technique with 6-0 Prolene sutures (Ethicon, Inc, Sommerville, NJ; Figure 2). The anastomosis of the bronchus was constructed by using an end-to-end suturing technique with 4-0 Prolene sutures. The anastomosis of the pulmonary artery was performed by using an end-to-end suturing technique with 6-0 Prolene sutures. The graft was covered with sponges and ice slush during implantation to avoid warm ischemic injury. Before reperfusion, methylprednisolone $(5 \mathrm{mg} /$ $\mathrm{kg}$ ) was administered intravenously. After the vascular clamps were removed, all anastomoses were checked for air or blood leakage. After closing the right chest, the animal was placed in the right lateral decubitus position. A left thoracotomy was performed through the fifth intercostal space. The left lower lobe was removed, and the left upper and middle lobes were spared. The left graft (left lower lobe of the donor) was implanted in the natural anatomic position in the same manner as for the right graft (Figures 1 and 2). 


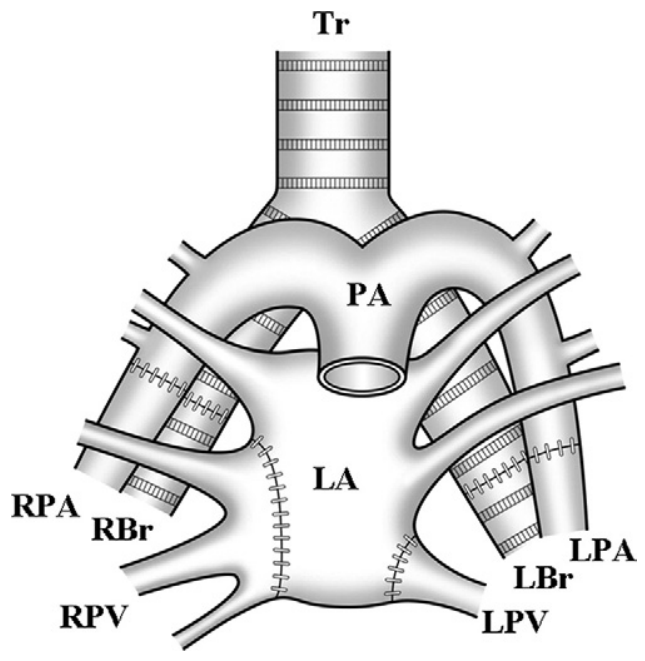

Figure 2. Alignment of the 2 donor grafts with the recipient hilum. $T r$, Trachea; $P A$, pulmonary artery; $L A$, left atrium; $R P A$, right pulmonary artery; $R B r$, right bronchus; $R P V$, right pulmonary vein; $L P A$, left pulmonary artery; $L B r$, left bronchus; $L P V$, left pulmonary vein.

\section{Acute Study}

Before thoracotomy, a $5 \mathrm{~F}$ thermodilution catheter was inserted through the right external jugular vein and positioned in the main pulmonary artery. Hemodynamic changes, including pulmonary artery pressure, central venous pressure, and cardiac output, were measured through this catheter by using a thermodilution method. A $5 \mathrm{~F}$ catheter was inserted into the left atrial appendage to measure left atrial pressure. Airway pressure was monitored by using a pressure transducer that was attached to the tracheal tube.

After transplantation, the recipient was placed in the supine position, with both chest incisions open. Both lungs were ventilated with a tidal volume of $20 \mathrm{~mL} / \mathrm{kg}$, a positive end-expiratory pressure of $5 \mathrm{~cm} \mathrm{H}_{2} \mathrm{O}$, and an inspired oxygen fraction of 1.0 at a rate of 15 breaths/min. Anesthesia was maintained with an intravenous infusion of thiopental sodium and vecuronium bromide. All pulmonary artery branches to the native spared lobes were ligated to allow all the cardiac output to flow to the transplanted grafts. The recipient dogs were observed for 3 hours, and aortic pressure, pulmonary artery pressure, central venous pressure, left atrial pressure, and airway pressure values were continuously recorded. Measurements of cardiac output and arterial blood gas analysis were performed at specific intervals (30, 60, 120, and 180 minutes after ligation of the pulmonary artery branches to the native spared lobes). Measurements were reported as the mean \pm standard error of the mean.

\section{Chronic Study}

After the chest closure, animals were immediately extubated once awake. The chest drainage tubes were removed about 1 hour postoperatively. The dogs received tacrolimus $(0.1 \mathrm{mg} / \mathrm{kg})$ intramuscularly and prednisone $(0.2 \mathrm{mg} / \mathrm{kg})$ orally every day. ${ }^{7}$ For 3 days postoperatively, cefazolin sodium $(500 \mathrm{mg}$ ) was injected intramuscularly, and heparin sodium (100 U/kg) was injected subcutaneously.

Three weeks after transplantation, surviving animals were reanesthetized and attached to a ventilator. Chest roentgenography was performed. A thermodilution catheter and a femoral artery line were inserted. Bilateral thoracotomy was performed through the fourth intercostal space, and the pulmonary artery branches to the native spared lobes were encircled with 1-0 silk ligatures. Function of the transplanted grafts was assessed under occlusion of all the pulmonary artery branches to the native spared lobes for 10 minutes. After the assessment, pulmonary angiography was performed. Then the animals were killed with an overdose of thiopental. Bronchial healing was assessed at autopsy.

\section{Animal Care}

All dogs received human care in compliance with the "Principles of laboratory animal care" formulated by the National Society for Medical Research and the "Guide for the care and use of laboratory animals" (http://www.nap.edu/catalog/5140.html).

\section{Results}

All 12 animals survived the operation. All 6 animals in the acute study survived the 3-hour assessment period on a ventilator. All 6 animals in the chronic study were successfully extubated. The ischemic time of the right graft was $124 \pm 9$ minutes, and that of the left graft was $265 \pm 15$ minutes.

\section{Acute Study}

The results of gas exchange and the hemodynamics of the recipients during the 3-hour assessment period are shown in Table 1. All 6 animals showed excellent lung and cardiac function throughout the observation period. At the end of the 3-hour assessment period, $\mathrm{PaO}_{2}$ was $589.0 \pm 14.4 \mathrm{~mm} \mathrm{Hg}$, and the mean pulmonary artery pressure was $35.8 \pm 3.9 \mathrm{~mm} \mathrm{Hg}$.

\section{Chronic Study}

The results of these 6 consecutive procedures are summarized in Table 2. Recipient dogs after extubation showed a normal respiratory pattern because the Herring-Breuer reflex for control of respiration was preserved by sparing native lobes.

One $\operatorname{dog}(\operatorname{dog} 8)$ died of pulmonary venous thrombus on postoperative day 5 . This animal had a small leakage at the atrial anastomosis in the right graft implantation that required the placement of a hemostatic stitch. At autopsy, this animal had a thrombus that originated at the site of repair. Bronchial healing of this dog was normal.

One $\operatorname{dog}$ (dog 12) experienced pneumothorax and required reoperation on postoperative day 4 . The source of the air leak was found in the interlobar fissure of the native spared right upper lobe. The air leak was repaired with fibrin glue, and this animal survived for 3 weeks without further complications.

Five (83\%) of 6 animals survived for 3 weeks. These 5 animals tolerated the occlusion of the pulmonary artery 
TABLE 1. Assessment of lung and cardiac function after transplantation

\begin{tabular}{|c|c|c|c|c|c|}
\hline & \multicolumn{4}{|c|}{ Acute study } & \multirow{2}{*}{$\frac{\text { Chronic study }}{3 \mathbf{w k}}$} \\
\hline & $30 \mathrm{~min}$ & $60 \mathrm{~min}$ & $120 \mathrm{~min}$ & $180 \mathrm{~min}$ & \\
\hline $\mathrm{PaO}_{2}(\mathrm{~mm} \mathrm{Hg})$ & $539.9 \pm 25.6$ & $554.1 \pm 23.8$ & $560.6 \pm 31.6$ & $589.0 \pm 14.4$ & $616.6 \pm 41.8$ \\
\hline Mean AoP (mm Hg) & $119.7 \pm 10.8$ & $114.5 \pm 11.1$ & $108.5 \pm 8.8$ & $106.5 \pm 9.6$ & $96.8 \pm 5.8$ \\
\hline Mean PAP $(\mathrm{mm} \mathrm{Hg})$ & $38.4 \pm 3.6$ & $33.8 \pm 3.2$ & $35.8 \pm 3.3$ & $35.8 \pm 3.9$ & $34.8 \pm 5.5$ \\
\hline Mean CVP (mm Hg) & $6.8 \pm 1.2$ & $5.9 \pm 1.2$ & $5.3 \pm 1.0$ & $5.8 \pm 0.8$ & $6.8 \pm 1.1$ \\
\hline PVR (dyne $\cdot \mathrm{s}^{-1} \cdot \mathrm{cm}^{-5}$ ) & $1826 \pm 347$ & $2110 \pm 257$ & $1706 \pm 295$ & $1831 \pm 423$ & $1580 \pm 304$ \\
\hline Mean AWP (mm Hg) & $5.3 \pm 0.3$ & $5.1 \pm 0.2$ & $5.3 \pm 0.3$ & $5.1 \pm 0.4$ & $4.8 \pm 0.1$ \\
\hline
\end{tabular}

All values are presented as means \pm standard error of the mean. Data were obtained after ligation of the pulmonary artery branches to the native spared lobes. AoP, Aortic pressure; PAP, pulmonary arterial pressure; CVP, central venous pressure; $C O$, cardiac output; $P V R$, pulmonary vascular resistance; $A W P$, airway pressure.

branches to the native spared lobes and showed excellent pulmonary function (Table 1). Chest roentgenograms showed well-expanded grafts without dead space (Figure 3). Pulmonary angiography demonstrated no stenosis in the arterial anastomoses (Figure 4). Good bronchial healing was observed in all dogs at autopsy (Table 2 and Figure 5).

\section{Discussion}

A great disparity between the supply of donor organs and the demand of potential recipients has resulted in longer waiting times and annual increases in deaths on the lung transplant waiting list. Bilateral LDLLT was developed as an alternative to cadaveric lung transplantation to circumvent this obstacle. ${ }^{1,2}$ In LDLLT right and left lower lobes from 2 healthy donors are implanted in the recipient in place of whole right and left lungs, respectively. Because a limited amount of lung tissue is implanted, this procedure is usually confined to patients of small size. Excessively small grafts might cause high pulmonary artery pressure, resulting in lung edema. ${ }^{8}$ A pleural space problem might increase the risk of empyema. ${ }^{9}$ Overexpansion of the donor lobes could contribute to obstructive physiology through early closure of the small airways. ${ }^{10}$ It is for this reason that large adult patients are thought to be poor candidates for LDLLT. Among 38 recipients receiving bilateral LDLLT in our institution, there were only 2 adult male patients. ${ }^{3,4} \mathrm{~A}$

TABLE 2. Results of chronic study

\begin{tabular}{|c|c|c|c|}
\hline & Survival (d) & Complications & $\begin{array}{c}\text { Bronchial } \\
\text { healing }\end{array}$ \\
\hline Dog 7 & 28 & None & Normal \\
\hline Dog 8 & 5 & Pulmonary venous thrombosis & Normal \\
\hline Dog 9 & 21 & None & Normal \\
\hline Dog 10 & 21 & None & Normal \\
\hline Dog 11 & 21 & None & Normal \\
\hline Dog 12 & 22 & Pneumothorax & Normal \\
\hline
\end{tabular}

number of patients have been turned down for LDLLT because of unacceptable size mismatch.

With the hope of expanding the possibilities for transplantation in large adult patients who are unlikely to survive the long wait for cadaveric lungs, we have developed a unique technique of UDLLT using a canine model. ${ }^{5,6}$ In UDLLT, 2 grafts obtained from donor animals were implanted in the right hemithorax after right pneumonectomy. One graft (left graft) was implanted as a right upper lobe in an upside-down position, and the other (right graft) was implanted in the natural anatomic position. UDLLT was technically possible and provided excellent early pulmonary

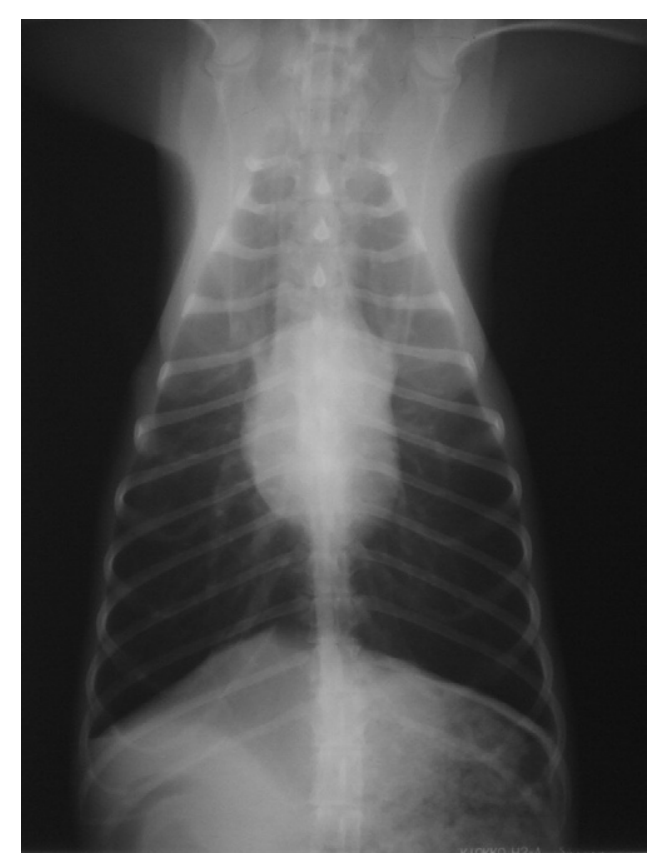

Figure 3. Chest roentgenogram of dog 9 three weeks after bilateral native lung-sparing lobar transplantation. Two grafts and native spare lobes fully expanded in both chest cavities. 


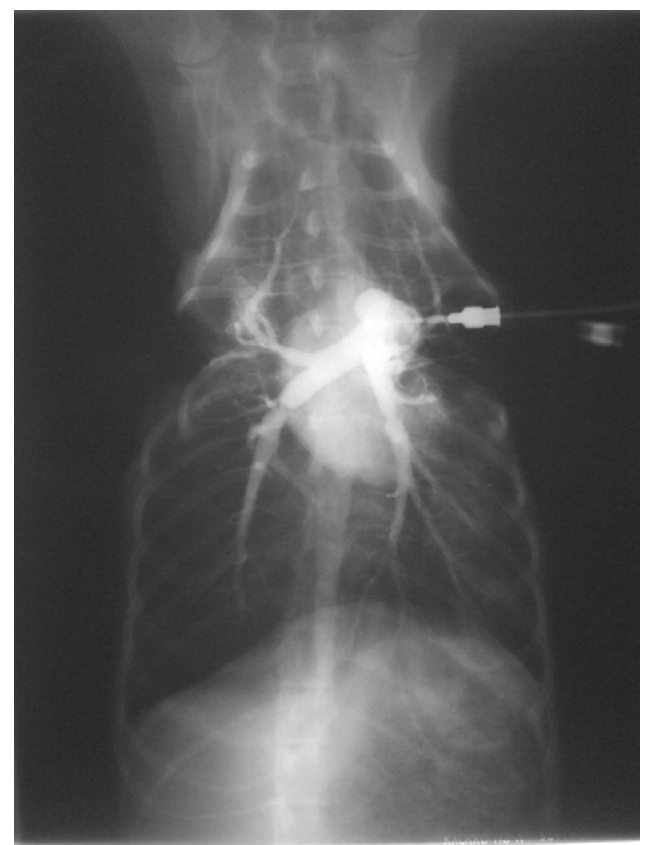

Figure 4. Pulmonary angiogram of dog 10 three weeks after bilateral native lung-sparing lobar transplantation. There was no stenosis in the arterial anastomoses.

function. ${ }^{5}$ However, it was associated with a relatively high incidence of vascular and bronchial complications. ${ }^{6}$ Of note was that these complications, such as pulmonary artery kinking, pulmonary venous thrombus, and bronchial dehiscence, occurred exclusively in the graft implanted in the upside-down position. To avoid the risk of complications related to the nonanatomically positioned graft, we developed a technique of BNSLT in which 2 grafts were implanted in the natural anatomic position.

We used a canine model to develop a technique of BNSLT. A single donor was used in this experiment to simplify the model. The right graft (right middle, lower, and cardiac lobes) resembles the human right lower lobe anatomically. The left graft (left lower lobe) resembles the human left lower lobe. Given the fact that the model has some difference from the human setting, such as chest shape and vascular anatomy, we believe that this model is good enough to develop a new technique of BNSLT.

BNSLT in human subjects, replacing both native lower lobes with 2 ipsilateral lower lobes, is based on the notion that single-lung transplantation has been successfully performed for various noninfectious lung diseases. ${ }^{11,12}$ The remaining native lobes, although they are diseased, provide some gas exchange capacity and vascular bed. Candidates for BNSLT should have no infection in the spared lobes and no pleural adhesion with well-developed interlobar fissures. Considering these factors, we propose that pulmonary fi-

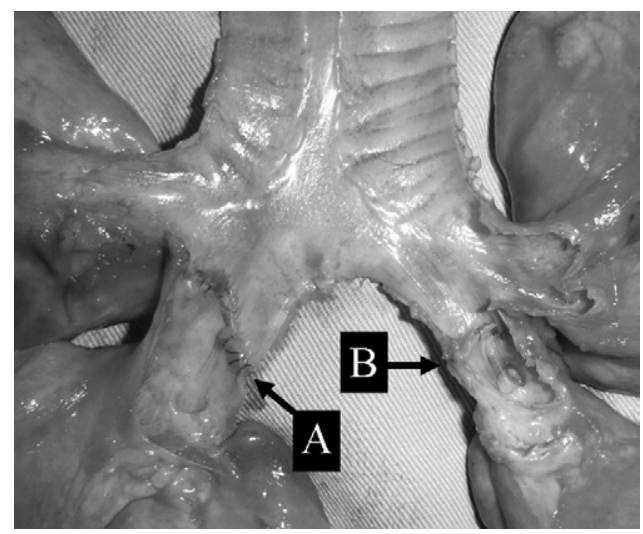

Figure 5. Excellent bronchial healing in dog 11 three weeks after bilateral native lung sparing lobar transplantation: $A$, intermediate bronchus; $B$, left lower bronchus.

brosis and primary pulmonary hypertension would be ideal indications for BNSLT. Pulmonary fibrosis with a history of pneumothorax would be a contraindication because of fused fissures with scarifications. Emphysema would not be a good indication because native lobe hyperinflation and persistent air leakage from the spared lobes could be potentially problematic. Because the number of available cadaveric donors is quite limited ( $<5$ cases per year in the whole country) in Japan, the only realistic option for most of our patients is to receive LDLLT and BNSLT, which would hopefully expand the possibilities for transplantation in large adult patients.

When the total forced vital capacity of the 2 grafts is more than $45 \%$ to $50 \%$ of the predicted forced vital capacity of the recipient, we accept the size disparity in LDLLT. ${ }^{3}$ When the ratio is less than $45 \%$, BNSLT would be indicated. In patients with restrictive disease, the acceptable ratio for BNSLT could be smaller than in patients with nonrestrictive disease because of a small chest cavity.

Several technical points required elaboration in BNSLT. Because the upper lobes were spared, working space was more limited, and all anastomoses were more distal compared with conventional lung transplantation. For both donor and recipient, lobectomies were to be performed to leave adequate length for subsequent vascular and bronchial anastomoses. The atrial anastomosis in the right graft implantation was the most difficult part of this operation. Care had to be taken in placing the vascular clamp across the recipient left atrium so as to avoid occlusion of the right upper and left lower pulmonary veins and to provide the maximal amount of tissue distal to the clamp for subsequent anastomosis. To prevent atrial venous thrombosis, we ensured intima-to-intima approximation and excluded intraluminal muscle by using a continuous everting mattress su- 
ture. We minimized peribronchial dissection and shortened the recipient bronchus to obtain good bronchial healing. Great care was taken to avoid any air leakage from the interlobar fissure.

With care of several technique points mentioned above, all anastomoses were easily aligned, and the implantation of both grafts did not present any difficulty. Morphologic adaptation of the 2 grafts was found to be excellent. Both grafts implanted in their anatomic positions filled the basal area of the bilateral thorax (Figure 3). The excellent early pulmonary function of the transplanted grafts was confirmed by ligating the pulmonary artery branches to the native spared lobes (Table 1).

In the chronic study pulmonary venous thrombus was encountered in one animal and resulted in lethal graft congestion. This animal had a small leakage at the atrial anastomosis in the right graft implantation that required the placement of a hemostatic stitch. At autopsy, this animal had a thrombus that originated at the site of repair. It has been well documented that thrombosis at the atrial suture line is a common complication in a canine lung transplantation model. ${ }^{13}$ In contrast, this complication is rare in human lung transplantation. ${ }^{14}$ The other animal had pneumothorax but was successfully managed by means of reoperation. The source of the air leak was found in the interlobar fissure of the native spared right upper lobe. We assume that these complications could have been avoided in the clinical setting. The satisfactory survival rate $(83 \%)$ with no bronchial complications and excellent pulmonary function in the chronic study was very encouraging (Tables 1 and 2). This experimental study suggested that BNSLT might be a viable new option for selected large adult patients who would not survive the long wait for cadaveric lungs.

We thank Tetsuo Kawakami for his expert technical assistance.

\section{References}

1. Starnes VA, Barr ML, Cohen RG, Hagen JA, Wells WJ, Horn MV, et al. Living-donor lobar lung transplantation experience. Intermediate results. J Thorac Cardiovasc Surg. 1996;112:1284-91.

2. Starnes VA, Bowdish ME, Woo MS, Barbers RG, Schenkel FA, Horn $\mathrm{MV}$, et al. A decade of living lobar lung transplantation. Recipient outcomes. J Thorac Cardiovasc Surg. 2004;127:114-22.

3. Date H, Aoe M, Nagahiro I, Sano Y, Andou A, Matsubara H, et al. Living-donor lobar lung transplantation for various lung diseases. J Thorac Cardiovasc Surg. 2003;126:476-81.

4. Date H, Aoe M, Sano Y, Nagahiro I, Miyaji K, Goto K, et al. Improved survival after living-donor lobar lung transplantation. J Thorac Cardiovasc Surg. 2004;128:933-40.

5. Okutani D, Date H, Hayama M, Inokawa H, Okazaki M, Nagahiro I, et al. The technique of unilateral double lobar lung transplantation in a canine model. J Thorac Cardiovasc Surg. 2004;127:563-7.

6. Aokage K, Date H, Sugimoto R, Okazaki M, Inokawa H, Okutani D, et al. A canine experimental study to assess the potential of unilateral double lobar lung transplantation. Eur J Cardiothorac Surg. 2006;29: 40-4.

7. Fukuse T, Hasegawa S, Hirai T, Hirata T, Muro K, Takahashi Y, et al. Optimal dose of FK 506 in canine lung transplantation. Transplant Proc. 1992;24:1399-400.

8. Fujita T, Date H, Ueda K, Nagahiro I, Aoe M, Andou A, et al. Experimental study on size matching in a canine living-donor lobar lung transplant model. J Thorac Cardiovasc Surg. 2002;123:104-9.

9. Backhus LM, Sievers EM, Schenkel FA, Barr ML, Cohen RG, Smith MA, et al. Pleural space problems after living lobar transplantation. J Heart Lung Transplant. 2005;24:2086-90.

10. Haddy SM, Bremner RM, Moore-Jefferies EW, Thangathurai D, Schenkel FA, Barr ML, et al. Hyperinflation resulting in hemodynamic collapse following living donor lobar transplantation. Anesthesiology. 2002;97:1315-7.

11. Trulock EP, Edwards LB, Taylor DO, Boucek MM, Keck BM, Hertz MI. Registry of the International Society for Heart and Lung Transplantation: twenty-second official adult lung and heart-lung transplant report-2005. J Heart Lung Transplant. 2005;24:956-67.

12. Meyers BF, Lynch JP, Trulock EP, Guthrie T, Cooper JD, Patterson GA. Single versus bilateral lung transplantation for idiopathic pulmonary fibrosis: a ten-year institutional experience. $J$ Thorac Cardiovasc Surg. 2000;120:99-107.

13. Stevens GH, Rangel DM, Yakeishi Y, Fonkalsrud EW. Technical problems in lung allotransplantation. Causes of pulmonary venous outflow obstruction. Arch Surg. 1969;99:506-12.

14. Nagahiro I, Horton M, Wilson M, Bennetts J, Spratt P, Glanville AR. Pulmonary vein thrombosis treated successfully by thrombectomy after bilateral sequential lung transplantation: report of a case. Surg Today. 2003;33:82-4. 